
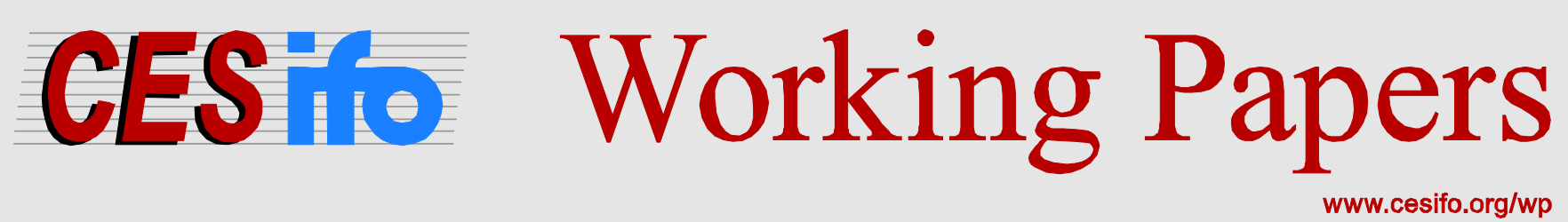

\title{
The Gini Coefficient and Personal Inequality Measurement
}

\author{
James B. Davies
}

CESIFO WORKING PAPER NO. 5961

CATEgORY 1: Public FinANCE

ORIGINAL VERSION: JUNE 2016

THIS VERSION: JANUARY 2017

An electronic version of the paper may be downloaded

- from the SSRN website:

- from the RePEc website:

- from the CESifo website:

wWw.SSRN.com

Www.RePEc.org

www.CESifo-group.org/wp 


\title{
The Gini Coefficient and Personal Inequality Measurement
}

\begin{abstract}
The Gini coefficient is based on the sum of pairwise income differences. For an individual, differences vis-à-vis poorer people represent advantage, and those versus richer people deprivation. Any weighted average of deprivation and advantage generates a "Gini admissible" personal inequality index. The mean value of such an index across individuals equals the Gini coefficient. Properties of the personal indexes explain the differing sensitivity of the Gini coefficient to inequality in various ranges of the income distribution. Applications to the Kuznets transformation in developing countries, to polarization in advanced countries and to broad increases or decreases in income dispersion are explored.
\end{abstract}

JEL-Codes: D300, D630.

James B. Davies

Department of Economics

University of Western Ontario

Canada - London, N6A 5C2

jdavies@uwo.ca

January, 2017

I would like to thank Stephen Gordon, Michael Hoy, Stephen Jenkins, Gregory Pavlov, Shlomo Yitzhaki, and participants in a seminar at the University of Manitoba for comments and suggestions. Responsibility for any errors or omissions is mine. 


\section{Introduction}

The Gini coefficient has a natural interpretation as the mean of personal inequality assessments. While that fact is obvious once pointed out, it was not emphasized in the original work by Gini (1914) and has not been highlighted since. This paper shows that this straightforward interpretation throws important light on the properties of the Gini coefficient. It also allows us to better understand the reaction of the Gini coefficient, and possibly also that of individuals, to secular changes in income distribution. The latter include the transition from a traditional to a modern economy analyzed by Kuznets (1955), and the polarization and rising inequality seen in recent decades in the US and many other countries. Personal assessments of the direction of change in inequality may differ between people at different income levels. These results suggest that our understanding of inequality measurement can be enriched by studying what it may mean at the personal level.

The Gini coefficient can be defined or interpreted in many ways (Yitzhaki, 1998). For our purposes the most useful is that it equals one half the mean difference divided by the mean. That is, the Gini coefficient can be found by taking the sum of all pairwise absolute income differences, $S$, converting to an average and normalizing by the mean income. $S$ equals the sum across individuals $i=1, \ldots, n$ of their personal sums of income differences with all other individuals, $S_{i}$. The latter provide the basis for a personal inequality index whose average across the population is the Gini coefficient.

For each individual, $S_{i}$ is composed of the sum of differences with respect to people at higher incomes plus the sum of differences vis- à -vis lower income persons. Following Yitzhaki (1979) the sum of differences with higher incomes may be used to define the individual's deprivation. That concept is complemented by the individual's advantage, derived from the sum of differences with respect to lower incomes. ${ }^{1}$ Summing either deprivation or advantage across the whole population produces the same total (Yitzhaki, 1979). Therefore, any weighted average of deprivation and advantage, as well as an unweighted average, will generate the Gini coefficient. This means that there is a whole family of "Gini admissible" personal inequality indexes or GAPIIs. One implication is that if societies choose to base overall inequality measurement on an average of individual assessments they may all use the Gini coefficient at the aggregate level even if they differ in the weight placed on advantage vs. deprivation at the personal level.

The personal inequality indexes discussed here have both "top down" and "bottom up" interpretations. This paper does not take a position on which viewpoint is preferable. It is not necessary to make a choice in order to pursue the analysis. A GAPII could be regarded as reflecting how a social planner would measure personal inequality. This is a "top down" interpretation. The "bottom up" view is that it would be reasonable for individuals themselves to assess inequality using a GAPII. Why might they do so? One possibility is that they could have interdependent utility functions that suggest the use of a GAPII (Fehr and Schmidt, 1999). Another may lie in bounded rationality. Holding the mean constant, in a

\footnotetext{
${ }^{1}$ Yitzhaki (1979) used the term "relative deprivation", which was introduced by Runciman (1966) to refer to any case in which some members of a reference group felt deprived compared to other members of their group. "Deprivation" is used here simply because it is shorter. Fehr and Schmidt (1999) referred to the same concept as "disadvantageous inequality", but the term deprivation still dominates in the literature. Yitzhaki (1979) used the term "satisfaction" rather than "advantage". "Advantage" is used here as a more neutral term.
} 
society of two people the difference between their incomes is a natural indicator of inequality. People might, implicitly if not explicitly, extend this to regard the average of pairwise differences as an attractive indicator of inequality when there are more than two people. That conclusion could be reinforced by information and computational constraints. As shown in this paper, in order to compute the value of a GAPII the individual only needs to know the fractions of the population with income above and below her and the average incomes of those two groups. While we should not suppose that real-world individuals know everyone else's income, they might be able to make a serviceable guess at these fractions and averages.

If the "bottom up" interpretation of GAPIIs is taken, our analysis is clearly related to the literature on individual attitudes toward inequality. A portion of that literature attempts to measure attitudes within narrow reference groups, e.g. co-workers or members of the same occupation. In such cases people tend to be averse to deprivation but to like advantage. As Clark and D'Ambrosio (2015) point out, in the income distribution literature the usual reference group is broader. In that context, following Yitzhaki $(1979,1982)$ and Fehr and Schmidt (1999) the general expectation has been that people will be averse to both deprivation and advantage. There are now a few empirical and experimental studies that have estimated aversion to deprivation and/or advantage with broader reference groups. Using the German SOEP survey data, D'Ambrosio and Frick (2007) find strong aversion to deprivation (but do not report on attitudes to advantage). Cojocaru (2014) finds significant aversion to both advantage and deprivation using a survey of 27 transition countries. In experiments with subjects who played a sequential public goods game, Teyssier (2012) found that $40 \%$ were averse to both advantage and deprivation while $18 \%$ were averse to neither. While these studies do not provide strong evidence on the relative degree of aversion to deprivation vs. advantage, neural studies find that brain activity reacts more strongly to deprivation (Clark and D'Ambrosio, 2015) and there appears to be close to a consensus that aversion is stronger to deprivation than to advantage for most people.

The remainder of the paper proceeds as follows. For expositional simplicity we start by working with the case in which advantage and deprivation are equally weighted. Section II defines the personal inequality index and derives some of its basic properties. In Section III we then explore how the behavior of this index helps to explain the sensitivity of the Gini coefficient to income changes in different ranges of a distribution. The analysis is extended to allow unequal weighting of deprivation and advantage in Section IV, which shows that the main insights of the previous two sections survive this generalization. How the personal assessments of inequality vary with income is discussed in Section $\mathrm{V}$ and the behavior of those assessments during periods of secular change in income distribution is examined in Section VI. Section VII concludes.

\section{Gini-admissible Personal Inequality Indexes: Base Case}

In this section we see how the Gini coefficient can be defined as the average value across individuals of a particular personal inequality index (PII), and begin to examine the properties of the latter. This index is an example of a "Gini admissible" PII, or GAPII. A PII qualifies as a GAPII if its simple average across individuals equals the Gini coefficient. 
The Gini coefficient for an income distribution equals one half the mean difference divided by the mean, as in:

$$
G=\frac{1}{2 n^{2} \bar{y}} \sum_{i=1}^{n} \sum_{j=1}^{n}\left|y_{i}-y_{j}\right|=\frac{S}{2 n^{2} \bar{y}}
$$

where $y_{i}$ is the income of individual $i, \bar{y}$ is mean income, $n>1, y_{1} \leq y_{2} \leq \cdots \leq y_{n}, S$ is the sum of differences, and $S / n^{2}$ is the mean difference. ${ }^{2}$

A natural but previously overlooked interpretation is that $G$ is the mean value across individuals of a particular GAPII, $G_{i}$ :

$$
G=\frac{1}{n} \sum_{i=1}^{n} G_{i}
$$

where

$$
G_{i}=\frac{1}{2 n \bar{y}} \sum_{j=1}^{n}\left|y_{i}-y_{j}\right|=\frac{S_{i}}{2 n \bar{y}}
$$

and $S_{i}$ is the sum of differences for individual $i$. Equation (3) can be rewritten:

$$
G_{i}=\frac{1}{2 n \bar{y}}\left[n_{i}^{l}\left(y_{i}-\bar{y}_{i}^{l}\right)+n_{i}^{h}\left(\bar{y}_{i}^{h}-y_{i}\right)\right]
$$

where $n_{i}^{l}$ is the number of individuals with income less than or equal to $y_{i}$, excluding individual $i$, and $n_{i}^{h}$ is the number with income strictly greater than $y_{i}$, so that $n_{i}^{l}+n_{i}^{h}=n-1 .^{3} \quad \bar{y}_{i}^{l}$ and $\bar{y}_{i}^{h}$ are mean income among those with income less than or equal to $y_{i}$, excluding $i$, and strictly greater than $y_{i}$ respectively.

Let $H_{i}$ be the set of all $j$ such that $y_{j}>y_{i}$, and $L_{i}$ be the set of all $j$ excluding $i$ such that $y_{j} \leq y_{i}$. Equation (4) can be expressed as:

$$
G_{i}=\frac{1}{2 \bar{y}}\left(A_{i}+D_{i}\right)
$$

where:

$$
A_{i}=\frac{n_{i}^{l}}{n}\left(y_{i}-\bar{y}_{i}^{l}\right)=\frac{1}{n} \sum_{j \in L_{i}}\left(y_{i}-y_{j}\right)
$$

\footnotetext{
${ }^{2}$ As mentioned earlier, the Gini coefficient can be expressed in many different ways (Yitzhaki, 1998). This is one of the two principal forms in which it was originally set out in Gini (1914), and is the most convenient for our discussion.

${ }^{3}$ The choice to include individuals who have the same income as $i$ in the lower group rather than in the higher group is arbitrary but does not affect the results in any significant way.
} 


$$
D_{i}=\frac{n_{i}^{h}}{n}\left(\bar{y}_{i}^{h}-y_{i}\right)=\frac{1}{n} \sum_{j \in H_{i}}\left(y_{j}-y_{i}\right)
$$

$D_{i}$ is the discrete analogue of the measure of relative deprivation for an individual, which we will refer to simply as deprivation, proposed by Yitzhaki (1979) for a continuous distribution. It equals the average shortfall of $i$ 's income below the income of those who are better off, weighted by the fraction of the population in the latter group. Equation (4') shows that $G_{i}$ is the simple average of $D_{i}$ and a complementary measure, $A_{i}$, normalized by the mean. We will say that $A_{i}$ represents individual $i$ 's advantage compared to people with lower income. Thus from the individual perspective inequality consists of both deprivation with respect to the better off and advantage over the worse off.

While $G_{i}$ is a natural personal inequality index to associate with the Gini coefficient, it is not the only GAPII. As mentioned earlier, and as shown in Section IV, one can define a more general class of GAPIIs that are based on a weighted average of $A_{i}$ and $D_{i} . G_{i}$ is a special case in which the weights on $A_{i}$ and $D_{i}$ are equal.

From (4) we have:

Proposition 1: $G_{i}$ is insensitive to a transfer of income within $H_{i}$ or within $L_{i}$ if the composition of neither group changes as a result of the transfer.

The proposition follows from the fact that transfers of income confined either to $H_{i}$ or $L_{i}$ do not alter $n_{i}^{l}, \bar{y}_{i}^{l}, n_{i}^{h}$, or $\bar{y}_{i}^{h}$ or any other term on the right-hand side of (4). In terms of (4'), as noted by Yitzhaki (1979) these transfers have no effect on advantage, $A_{i}$, or on deprivation, $D_{i}$. The insensitivity of $G_{i}$ to such transfers means that it does not respect the Pigou-Dalton principle of transfers, which is a cornerstone of the theory of aggregate inequality measurement. ${ }^{4}$ That an aggregate index that respects the Pigou-Dalton principle can be built on the basis of personal indexes that violate the principle is striking.

Sensitivity of $G_{i}$ to a transfer of income between $H_{i}$ and $L_{i}$

What determines how sensitive $G_{i}$ is to a transfer of income between $H_{i}$ and $L_{i}$ ? Consider the transfer of a total amount $R$ from $H_{i}$ to $L_{i}$. Note that such a transfer reduces both $A_{i}$ and $D_{i}$ by $R / n$, as can be seen from (5) where $n_{i}^{l}\left(y_{i}-\bar{y}_{i}^{l}\right)$ and $n_{i}^{h}\left(\bar{y}_{i}^{h}-y_{i}\right)$ both fall by $R$. We will allow $R$ to be negative, so this also handles the case of transfers from $L_{i}$ to $H_{i}$, which increase $A_{i}$ and $D_{i}$ by equal amounts. Using

$$
\frac{\partial A_{i}}{\partial R}=\frac{\partial D_{i}}{\partial R}=\frac{-1}{n}
$$

from (4') we have:

(6) $\frac{\partial G_{i}}{\partial R}=-\frac{1}{n \bar{y}}$

\footnotetext{
${ }^{4}$ Dalton (1920, p. 351) identified the central importance of the "principle of transfers", which says that a rankpreserving transfer from a richer person to a poorer person reduces inequality. Dalton referred his readers to an earlier statement of the same idea by Pigou. More recently the principle has come to be referred to as the "PigouDalton" principle of transfers (Sen, 1973).
} 
which allows us to state:

Proposition 2: When income is transferred from a person with income strictly above $y_{i}$ to someone with income strictly below $y_{i}, G_{i}$ falls, while if income is transferred from a person with income strictly below $y_{i}$ to someone with income strictly above $y_{i}, G_{i}$ rises. In both cases the change in $G_{i}$ is proportional to the amount transferred and independent of $y_{i}$.

Sensitivity of $G_{i}$ to a transfer affecting $y_{i}$

We also need to analyze those cases where distributional changes affect individual $i$ 's own income. There are two situations to consider. One is that of a transfer from $i$ to another person $j$. The other is that of a transfer from $j$ to $i$. We will consider them in turn. In this analysis, and in the remainder of the paper unless indicated otherwise, we will assume $y_{1}<y_{2}<\cdots<y_{n}$. This assumption will simplify the analysis since, for example, it implies that when $n$ is odd there is a unique individual with median income, $y^{\text {med }}$, and half the remaining population has $y_{i}<y^{\text {med }}$ while the other half have $y_{i}>y^{\text {med }} .{ }^{5}$ If $n$ is even there is no individual with $y_{i}=y^{\text {med }}$, but $y^{\text {med }}$, which is defined as the midpoint between $y_{n / 2}$ and $y_{n / 2+1}$, again divides the population into two sub-populations of equal size with incomes above and below the median.

Transfer from $i$ to $j$ : Let $y_{i}^{o}$ and $y_{j}^{o}$ be initial incomes and consider the effect on $G_{i}$ of the transfer of a small amount $r$ from individual $i$ to individual $j$. From (4) we obtain:

Proposition 3a: The effect on $G_{i}$ of a small transfer in the amount of $r$ from individual $i$ to an individual $j$ is given by:

$$
\begin{aligned}
\Delta G_{i} & =\frac{1}{2 n \bar{y}}\left[\left(n_{i}^{h}-n_{i}^{l}\right)-1\right] \mathrm{r}, \quad i>j \\
\Delta G_{i} & =\frac{1}{2 n \bar{y}}\left[\left(n_{i}^{h}-n_{i}^{l}\right)+1\right] \mathrm{r}, \quad i<j
\end{aligned}
$$

If we could ignore the -1 and +1 in the square brackets on the right-hand side, (7) would say that irrespective of whether $i$ was greater or less than $j$, a transfer from $i$ to anyone else would increase $G_{i}$ if $i$ was below the median and reduce $G_{i}$ if $i$ was above the median. This reflects the fact that the main impact of the transfer on $G_{i}$ is to reduce $A_{i}$ and increase $D_{i}$. If $n_{i}^{h}>n_{i}^{l}$, individual $i$ is below the median and from (5) we see that the increase in $D_{i}$ will exceed the drop in $A_{i}$, since those changes are proportional to $n_{i}^{h}$ and $n_{i}^{l}$ respectively. If $n_{i}^{h}<n_{i}^{l}$, individual $i$ is above the median and we have the opposite case. The -1 in (7i) means that the rank at which $\Delta G_{i}$ switches from being positive to negative as we go up the income scale in the $i>j$ case is one position higher than it would otherwise be, since the transfer is going to a person with income lower than the "donor" $i$, which reduces $\bar{y}_{i}^{l}$ and $A_{i}$ a little. And

\footnotetext{
${ }^{5}$ If we assume only $y_{1} \leq y_{2} \leq \cdots \leq y_{n}$ then there could be multiple individuals with median income and the groups with income strictly below the median and strictly above the median need not contain an equal number of members. Consider for example a population with the set of incomes $(1,1,2,2,2,3)$.
} 
the +1 in (7ii) means that when $i<j, \Delta G_{i}$ switches from positive to negative one position lower than would otherwise be the case since the transfer goes to a higher income person, raising $\bar{y}_{i}^{h}$ and $D_{i}$ a little.

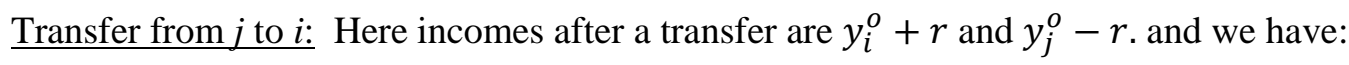

Proposition 3b: The effect on $G_{i}$ of a small transfer in the amount of $r$ from an individual $j$ to individual $i$ is given by:

$$
\begin{aligned}
\Delta G_{i} & =\frac{1}{2 n \bar{y}}\left[\left(n_{i}^{l}-n_{i}^{h}\right)+1\right] \mathrm{r}, \quad i>j \\
\Delta G_{i} & =\frac{1}{2 n \bar{y}}\left[\left(n_{i}^{l}-n_{i}^{h}\right)-1\right] \mathrm{r}, \quad i<j
\end{aligned}
$$

Now the main effect of the transfer is to raise $y_{i}$ and therefore to increase $A_{i}$ and reduce $D_{i}$, which is equalizing if $y_{i}$ is below the median and disequalizing if $y_{i}$ is above the median. Again the point at which $\Delta G_{i}$ switches sign as $i$ rises is offset one position by the small impact of the change in $y_{j}$ on $A_{i}$ when $i>j$ and on $D_{i}$ when $i<j$.

Summing up, we can say that for an individual whose income is above the median, a small transfer from herself to someone else is equalizing, from a personal standpoint, if her income is above the median, and is disequalizing if her income is below the median, subject to the small qualification evident in (7i, 7ii). If she is the recipient a small transfer is equalizing from the personal viewpoint if she is below the median and disequalizing if she is above the median, again subject to a small qualification (see 8i, 8ii). Thus the situation in Gini-admissible personal inequality measurement is quite different from that in the familiar aggregate inequality measurement. In the latter, the impact of a small transfer on inequality is deemed equalizing if the donor's income exceeds the recipient's and disequalizing if the opposite holds. In the case of Gini-admissible personal inequality measurement, in contrast, whether the transfer is considered equalizing or disequalizing depends almost solely on the income of the person for whom the assessment is being made. For low income people, if they make a transfer it is disequalizing while if they receive a transfer it is equalizing. For high income people the opposite holds..

\section{Explaining the sensitivity of the Gini coefficient to changes in different ranges of the income distribution}

From (1) one may derive:

$$
G=\frac{2}{n^{2} \bar{y}}\left[y_{1}+2 y_{2}+3 y_{3}+\cdots+n y_{n}\right]-\frac{n+1}{n}
$$

(see e.g. Cowell, 2011, p. 114). This provides insight into the sensitivity of the Gini coefficient to changes in different ranges of the income distribution. Consider a small transfer, $r$, from individual $j$ to individual $i$ where $i<j$. This is an example of what would be called an "equalizing transfer" in discussions of aggregate inequality. From (9), this transfer will produce a change in the Gini coefficient given by: 


$$
\Delta G=\frac{-2 r(j-i)}{n^{2} \bar{y}}
$$

which also tells us the impact of a transfer from $i$ to $j$, in which case $r<0$. We see that the impact on the Gini coefficient does not depend on $y_{i}$ or $y_{j}$, but varies only with $r$ and the difference in income ranks between $i$ and $j$.

The fact that the sensitivity of the Gini coefficient to transfers is independent of the incomes of the transferor and transferee, but depends on the number of people between them in the distribution, is one of the most interesting properties of the Gini coefficient. This property follows directly from those of the personal inequality index $G_{i}$ captured in Propositions 1, 2 and 3 above. Again considering a small transfer, $r$, from individual $j$ to individual $i$ where $i<j$, Proposition 1 implies:

$$
\Delta G_{k}=0 . \quad k<i, k>j .
$$

From Proposition 2 we have:

$$
\Delta G_{k}=\frac{-r}{n \bar{y}} . \quad i<k<j .
$$

And from Proposition 3

$$
\Delta G_{i}=\frac{\left(n_{i}^{l}-n_{i}^{h}-1\right) r}{2 n \bar{y}} . \quad \Delta G_{j}=\frac{\left(n_{j}^{h}-n_{j}^{l}-1\right) r}{2 n \bar{y}} .
$$

Now, from (2) and (11i), the change in $G$ resulting from a transfer from $j$ to $i$ is given by:

$$
\Delta G=\frac{1}{n}\left(\Delta G_{i}+\Delta G_{j}+\sum_{k=i+1}^{j-1} \Delta G_{k}\right)
$$

Note first from (11ii) that

$$
\sum_{k=i+1}^{j-1} \Delta G_{k}=-(j-i-1) \frac{r}{n \bar{y}}
$$

which is proportional to the number of people between $i$ and $j$, that is the number of people the transfer from $j$ to $i$ "passes over".

Next, to complete the analysis of $\Delta G$, note from (12) that:

$$
\begin{aligned}
\Delta G_{i}+\Delta G_{j} & =\frac{\left(n_{i}^{l}-n_{i}^{h}-1\right) r}{2 n \bar{y}}+\frac{\left(n_{j}^{h}-n_{j}^{l}-1\right) r}{2 n \bar{y}} \\
& =\frac{-r}{2 n \bar{y}}\left[\left(n_{j}^{l}-n_{i}^{l}\right)+\left(n_{i}^{h}-n_{j}^{h}\right)+2\right]
\end{aligned}
$$

Since $n_{j}^{l}-n_{i}^{l}$ and $n_{i}^{h}-n_{j}^{h}$ both equal $j-i$ we have: 


$$
\Delta G_{i}+\Delta G_{j}=\frac{-r}{n \bar{y}}(j-i+1)
$$

Hence, like $\sum_{k=i+1}^{j-1} \Delta G_{k}, \Delta G_{i}+\Delta G_{j}$ is proportional to the size of the transfer and rises linearly, in absolute value, with the number of people between $i$ and $j .{ }^{6}$ In this case the reason for dependence on the number of people between $i$ and $j$ is that the effects of the transfer cancel out for $A_{i}$ and $A_{j}$ on the one hand, and for $D_{i}$ and $D_{j}$ on the other, where the sums they are based on overlap. The range of overlap includes all $k<i$ for $A_{i}$ and $A_{j}$, and all $k>j$ for $D_{i}$ and $D_{j}$. The range where effects do not cancel out has $j-i+1$ people in it.

Summing up, substituting (14) and (15) into (13) we have:

$$
\Delta G=\frac{-r}{n^{2} \bar{y}}[(j-i+1)+(j-i-1)]=\frac{-2 r(j-i)}{n^{2} \bar{y}}
$$

which is the same as (10). So we have shown that the mean of the effects on the personal inequality indexes resulting from the transfer equals the change in $G$ that one would expect from aggregate inequality analysis.

The purpose of this exercise has been to show that the effects of a transfer on personal inequality explain the impact on $G$. That the reaction of $G$ is governed by the number of people between transferor $j$ and transferee $i$ is due to two things: (i) aside from $i$ and $j$ themselves, the only people whose personal inequality is affected by the transfer are the individuals between them in the distribution, and (ii) the effects of the transfer on $G_{i}$ and $G_{j}$ cancel out except for those based on changes in income gaps between $i$ or $j$ and individuals in the range $(i+1, j-1)$.

\section{Unequal Weighting of Deprivation and Advantage}

Yitzhaki (1979) defined relative deprivation for a society as a whole, $D$, as the average of individual deprivation indexes $D_{i}$. He worked with continuous distributions. The corresponding relationship with a discrete income distribution is:

$$
D=\frac{1}{n} \sum_{i=1}^{n} D_{i}
$$

We can define overall advantage in a parallel way as:

$$
A=\frac{1}{n} \sum_{i=1}^{n} A_{i}
$$

Yitzhaki shows that $D$ is related to the Gini coefficient according to:

\footnotetext{
${ }^{6}$ Note that the right-hand-side of (15) is not proportional to the number of people between $i$ and $j$, which is $j-i-$ 1.
} 


$$
G=\frac{D}{\bar{y}}
$$

This result might appear puzzling, given that, from $\left(4^{\prime}\right), D_{i}$ represents only part of an individual's contribution to $G_{i}$ and therefore to $G$. The explanation is as follows. The Gini coefficient is proportional to the sum of differences, $S$. We can arrange the pairwise differences $\left|y_{i}-y_{j}\right|$ making up $S$ in a matrix $M$ with $i$ indexing rows and $j$ indexing columns. $D$ is the mean of the elements of $M$ above the main diagonal while $A$ is the mean of the below-diagonal elements. Now, the above-diagonal elements have the same mean as the below-diagonal elements in $M$, since e.g. $\left|y_{2}-y_{1}\right|=\left|y_{1}-y_{2}\right|$. Hence $A=D$. To get from $D$ to $S$ we must therefore double $D$ and multiply by $n^{2}$ (to go from an average to a sum). The same procedure could be used to generate $S$ from $A$. Thus we have $S=2 n^{2} D=2 n^{2} A$ or:

$$
A=D=\frac{S}{2 n^{2}}
$$

Substituting the expression for $D$ from (20) into (19) we obtain $G=S /\left(2 n^{2} \bar{y}\right)$, that is equation (1).

While Yitzhaki's approach and ours are closely related, his $D_{i}$ and our $G_{i}$ are distinct. $G_{i}$ depends not just on deprivation, $D_{i}$, but also on advantage, $A_{i}$. While, overall, $A=D$, at the individual level there is no such relationship. $A_{i}$ rises and $D_{i}$ falls as we move up through the income distribution from $y_{1}$ to $y_{n}$, and they do so at rates that rise or fall depending on the shape of the particular income distribution being examined.

The fact that $A=D$ has important consequences for our personal inequality indexes. Using (19) and $A=$ $D, G$ may be found by taking a weighted average of $A$ and $D$, as in:

$$
G=\frac{\lambda A+(1-\lambda) D}{\bar{y}} \quad 0 \leq \lambda \leq 1
$$

where we require the weights to be positive. This in turn reveals that there is a family of Gini admissible personal inequality indexes or GAPIIs of the form:

$$
G_{i}^{\lambda}=\frac{\lambda A_{i}+(1-\lambda) D_{i}}{\bar{y}} \quad 0 \leq \lambda \leq 1
$$

Hence, while $\lambda$ may differ across societies, they can nevertheless agree on using $G$ as an aggregate measure of inequality. In the continuous case this result could be generalized to allow $\lambda$ to differ across individuals, as long as the distribution of $\lambda$ was independent of individual income.

We may ask which of the results derived above for the $\lambda=1 / 2$ case survive once $\lambda \neq 1 / 2$ is allowed. Proposition 1, which says that the $G_{i}$ are insensitive to transfers entirely within the $H_{i}$ or $L_{i}$ comparator groups, survives. The principle is not affected by re-weighting income differences with the $H_{i}$ and $L_{i}$ groups via $\lambda \neq 1 / 2$. Proposition 2 , which says that when income is transferred from those with income above (below) $y_{i}$ to those with income below (above) $y_{i}$ the fall (rise) in $G_{i}$ is proportional to the total amount transferred, $R$, and is independent of $y_{i}$ is also unaltered because we still have:

$$
\frac{\partial A_{i}}{\partial R}=\frac{\partial D_{i}}{\partial R}=\frac{-1}{n}
$$


and (6) survives unchanged because in the more general formulation, using (22) we have:

$$
\frac{\partial G_{i}^{\lambda}}{\partial R}=\frac{1}{\bar{y}}\left[\lambda \frac{\partial A_{i}}{\partial R}+(1-\lambda) \frac{\partial D_{i}}{\partial R}\right]=-\frac{1}{n \bar{y}}
$$

Proposition 3 described the impact on $G_{i}$ of making a small transfer from another person to individual $i$. Assuming $y_{1}<y_{2}<\cdots<y_{n}$, the conclusion in the $\lambda=1 / 2$ case was that, except for a very small region around the median, a transfer from a higher income person would reduce $G_{i}$ if $y_{i}$ was below the median, and increase $G_{i}$ if $y_{i}$ was above the median. Converse results held if the transfer came from a lower income person. The critical role of the median arose because with $\lambda=1 / 2$, advantage, $A_{i}$, and deprivation, $D_{i}$, are equally weighted. In general, the critical percentile is given by $1-\lambda$. Thus, for example, if one placed half as much weight on $A_{i}$ as on $D_{i}$, i.e. $\lambda=1 / 3$, the critical percentile would be $2 / 3$. That means that a small transfer from someone with higher income would be regarded as equalizing for or by almost everyone in the bottom two thirds of the population, but as disequalizing for almost all of those in the top third. This occurs because putting a higher weight on $D_{i}$ increases the equalizing impact on $G_{i}^{\lambda}$ from the fall in $D_{i}$ caused by such a transfer.

\section{Personal Inequality Assessments at Different Income Levels}

In this section we examine how $G_{i}^{\lambda}$ varies as $y_{i}$ rises from $y_{1}$ to $y_{n}$. We provide results for the general case where $\lambda$ can take on any value in the interval $[0,1]$, but note specific conclusions for the case where $\lambda=1 / 2$.

How does $G_{i}^{\lambda}$ change as we move up through the distribution of income? We continue to assume $y_{1}<$ $y_{2}<\cdots<y_{n}$. As we go from individual $i$ to $i+1$, the absolute income gaps in (3) or implicitly in (22) increase in value by $y_{i+1}-y_{i}$ for all $j$ such that $y_{j}<y_{i}$, and the corresponding gaps for all $j>i$ fall by the same amount. Hence we should expect that $G_{i}^{\lambda}$ will initially decline as $i$ rises from 1 , since at the start there are more people with $j>i$ than with $j \leq i$, until some critical point is reached, beyond which $G_{i}$ should begin to increase. Formally we have:

Proposition 4: If $y_{1}<y_{2}<\cdots<y_{n}$,

$$
\begin{aligned}
G_{i+1}^{\lambda} & >G_{i}^{\lambda} \text { as } \frac{i}{n}=1-\lambda . \\
& <
\end{aligned}
$$

\section{Proof: See Appendix.}

Proposition 4 indicates that $G_{i}^{\lambda}$ falls up to the $(1-\lambda) 100$ th percentile of the distribution and increases above that. As indicated above, this U-shaped pattern is based on the fact that moving from income $y_{i}$ to income $y_{i+1}$ increases the income gaps with lower income people and reduces those with higher income people by the same absolute amount. The relative impact of changes in the upper gaps compared with that of changes in the lower gaps is $(1-\lambda) / \lambda$. This means that $G_{i}^{\lambda}$ will fall more rapidly starting from $i=1$ if 
$\lambda<1 / 2$, compared with the $\lambda=1 / 2$ case, and less rapidly if $\lambda>1 / 2$. Note that if $\lambda=\frac{1}{2}, G_{i}^{\lambda}=G_{i}$ falls up to the $50^{\text {th }}$ percentile, that is up to the median, and rises thereafter.

We can also readily identify the value of $G_{i}^{\lambda}$ at the bottom and top of the distribution $(i=1$ and $i=n)$, as well as the value of $G_{i}^{\lambda}$ for the median individual, $G_{m e d}^{\lambda}$, if $n$ is odd. We have:

Proposition 5: If $y_{1}<y_{2}<\cdots<y_{n}$

(i) $G_{1}^{\lambda}=(1-\lambda)\left(1-\frac{y_{1}}{\bar{y}}\right)$

(ii) if $n$ is odd, $G_{m e d}^{\lambda}=\frac{n-1}{2 n \bar{y}}\left[(1-\lambda) \bar{y}_{m e d}^{h}-\lambda \bar{y}_{m e d}^{l}\right]$; if $n$ is even, $G_{m e d}^{\lambda}$ is not defined,

(iii) $G_{n}^{\lambda}=\lambda\left(\frac{y_{n}}{\bar{y}}-1\right)$

Proof: See Appendix.

Proposition 5 allows us to put upper bounds on $G_{1}^{\lambda}$ and $G_{n}^{\lambda}$. If $y_{1}$ is non-negative, the highest possible value of $G_{1}^{\lambda}$ is $1-\lambda$, which occurs when $y_{1}=0$. When deprivation and advantage are weighted equally, that is when $\lambda=\frac{1}{2}$, the maximum value is $\frac{1}{2}$. But the maximum value of $G_{1}^{\lambda}$ ranges from 0 , when $\lambda=1$ and personal inequality depends only on advantage, to 1 when $\lambda=0$ and it depends only on deprivation. In view of Proposition 4, these maxima also apply to all $G_{i}^{\lambda}$ up to the $(1-\lambda) 100$ th percentile. ${ }^{7}$ The upper bound on $G_{n}^{\lambda}$ occurs when one individual has all the income and $y_{n}=n \bar{y}$. In that case $G_{n}^{\lambda}=$ $\lambda(n-1)$, which is also an upper bound for all $G_{i}^{\lambda}$, s above the $(1-\lambda) 100$ th percentile.

Part (ii) of the proposition is also interesting, in throwing light on the value of the personal inequality index for the "average person", that is on the value of $G_{m e d}^{\lambda}$. The latter is based on a weighted average of $\bar{y}_{m e d}^{h}$ and $\bar{y}_{\text {med }}^{l}$, with the weight on $\bar{y}_{m e d}^{h}$ falling with $\lambda$. In the focal case with $\lambda=1 / 2$, we have:

$$
G_{m e d}=\frac{(n-1)}{4 n \bar{y}}\left(\bar{y}_{m e d}^{h}-\bar{y}_{m e d}^{l}\right)
$$

Since in any real-world example $(n-1) / n \approx 1$, this says:

$$
G_{\text {med }} \approx \frac{\bar{y}_{\text {med }}^{h}-\bar{y}_{\text {med }}^{l}}{4 \bar{y}}
$$

In the U.S. today, for household income before tax, $\bar{y}_{\text {med }}^{h} \approx \frac{8}{5} \bar{y}$ and $\bar{y}_{\text {med }}^{l} \approx \frac{2}{5}$, which yields $G_{m e d} \approx 0.3$, less than the value of the Gini coefficient, which was 0.476 in $2013 .{ }^{8}$ We may also note values of $G_{m e d}$

\footnotetext{
${ }^{7}$ Note that with $\lambda=1$, the $(1-\lambda) 100$ th percentile $=0$, so that $G_{i}^{\lambda}$ has no falling range.

${ }^{8}$ With the help of quintile share and other data from U.S. Census Bureau (2016) it can be estimated that $\bar{y}_{\text {med }}^{h}=$ $1.64 \bar{y}$ and $\bar{y}_{\text {med }}^{l}=0.36 \bar{y}$.
} 
under some familiar continuous distributions. $G_{m e d}$ would equal $\frac{1}{4}$ for a uniform distribution, and if $y_{i} \sim$ $N(\mu, \sigma)$, it would equal $\frac{2}{5} \frac{\sigma}{\mu}$, that is two-fifths of the coefficient of variation.

We can see that $G_{i}^{\lambda}$ will generally not be symmetric around the median. Looking at the $\lambda=1 / 2$ case again, for example, $G_{i}$ will never be greater than $1 / 2$ at the lowest income level, but can be very high at the top end. $G_{i}$ is not bounded above by 1 , unlike the Gini coefficient. $G_{n}=1$ is reached when $\frac{y_{n}}{\bar{y}}=3$. That ratio is exceeded in almost all real-world cases. This implies that, in a mathematical sense, from the standpoint of the rich there is more inequality than from that of the poor when $\lambda=1 / 2$, which is intuitive. For the rich there are relatively few people whose incomes is close to theirs, meaning there is a large gulf between their income and most others'.

\section{Personal Inequality During Secular Change in Income Distribution}

This section asks how $G_{i}^{\lambda}$ can be predicted to behave at different income levels during periods of secular change in income distribution. We focus initially in each case on the $\lambda=1 / 2$ case, in which deprivation and advantage are weighted equally, referring to $G_{i}^{1 / 2}$ simply as $G_{i}$, as above. We start with the Kuznets transformation and go on to the polarization and rising inequality that has been seen in the U.S. and many other high income countries in the last few decades. The principles at work are explored with the help of illustrative examples.

\section{Kuznets Transformation}

Kuznets (1955) studied what happens to income distribution and inequality in a growing economy where production is shifting from an initially large traditional agricultural sector to a modern sector. The modern sector eventually comprises most if not all of the economy. The consequences for inequality can be illustrated using a stylized model in which individual incomes are uniform within each of the sectors, higher in the modern sector, and unchanging during the growth process. ${ }^{9}$ (The assumption that individual incomes do not change within the sectors is relaxed below.) In this case the Gini coefficient, $G$, rises until the fraction of the population in the modern sector, $p$, hits a critical value, after which it declines. This critical value of $p$ is less than one half. That is because, while the mean difference has a maximum at $p=$ $1 / 2$, the mean, which appears in the denominator of the expression for $G$, is rising throughout, so $G$ has already started to decline at $p=1 / 2$.

The behavior of the GAPIIs, that is the $G_{i} \mathrm{~s}$, and $G$ during the Kuznets transformation will be illustrated here using an example whose implications are shown in Figure 1. It is assumed that income of each person in the traditional sector is $11.7 \%$ of per capita income in the modern sector. This gap is sufficient

\footnotetext{
${ }^{9}$ Kuznets considered a richer range of possibilities. He allowed unequal income distribution within both sectors and believed the leading case was one in which there was greater inequality in the modern sector than in the traditional, or agricultural, sector. He also considered the impacts of changes in the relative income, and of income inequality, in the modern vs. the agricultural sector over time. In most cases he found that as the relative population of the agricultural sector declined there was an initial increase in inequality followed by a decline.
} 


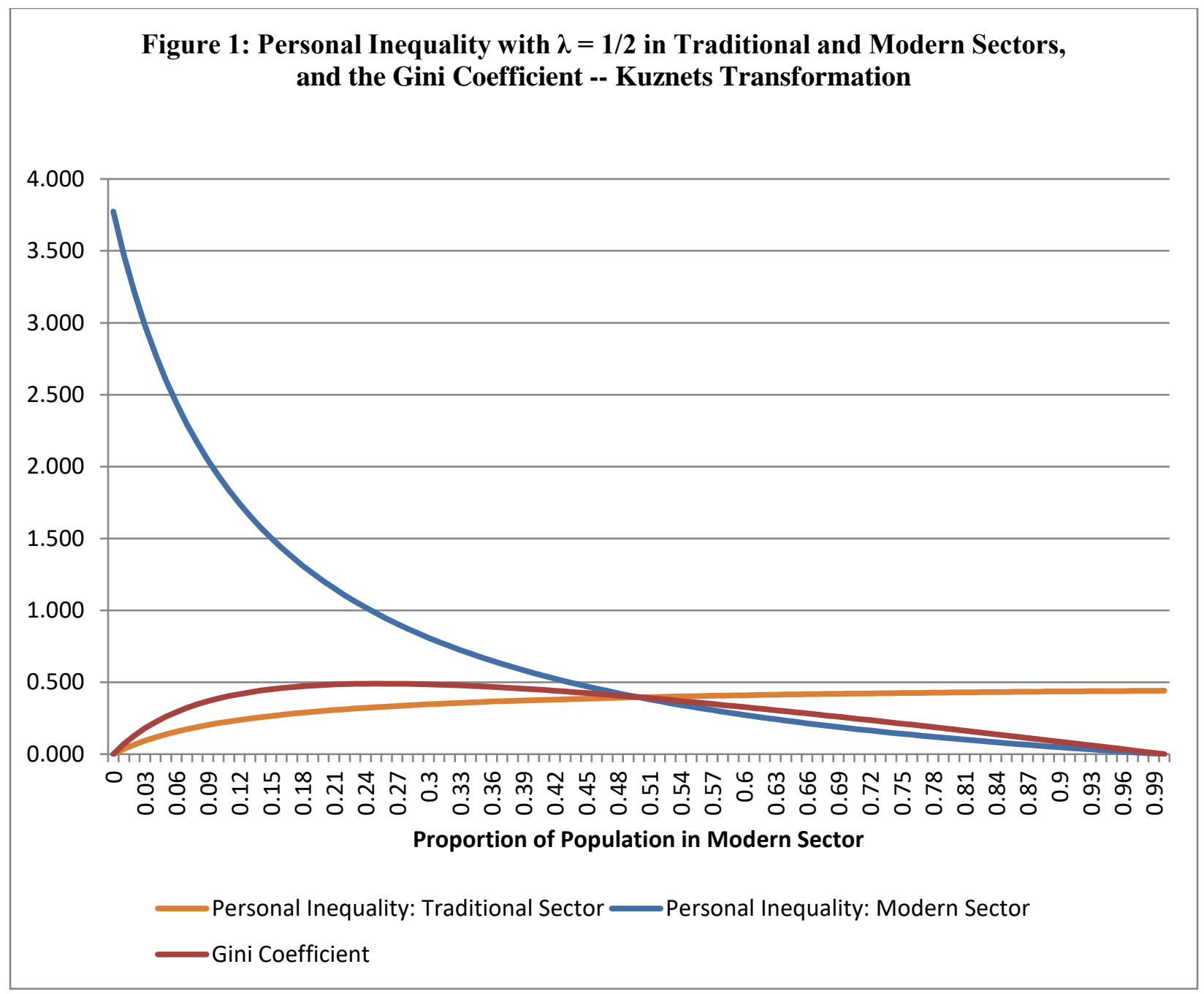

for the peak value of $G$ to be 0.49 , the value observed in China in 2008 ( $\mathrm{Li}$ and Sicular, 2014). China is the most prominent recent example of a society going through the kind of transformation that Kuznets described. In the early 1980s its Gini coefficient for family income fluctuated around 0.30 (Sicular, 2013). This was followed by a rapid rise with later deceleration to the 2008 peak, after which $G$ began to fall slowly. ${ }^{10}$ China may now be past a Kuznets curve peak. ${ }^{11}$

Taking the $\lambda=1 / 2$ case to begin with, we will refer to the individual inequality measures of people in the low and high income groups as $G_{L}$ and $G_{H}$ respectively. Since no one is worse off than those in the low income group, $G_{L}=\frac{D_{L}}{\bar{y}}$, that is it is based entirely on deprivation, while $G_{H}=\frac{A_{H}}{\bar{y}}$ and is based wholly on

${ }^{10}$ The National Bureau of Statistics estimates of the national Gini coefficient for family income were 0.491 in 2008 (Li and Sicular, 2014, Appendix A) and 0.469 in 2014 (Qi, 2015).

${ }^{11}$ Knight (2014) discusses whether China may be beyond the peak of the Kuznets curve. His conclusion is that this depends in part on public policy but that there are now strong underlying forces pushing in the direction of reducing inequality in China. 
advantage. Denoting income per person in the traditional sector $y_{L}$ and in the modern sector $y_{H}$, and adopting corresponding notation for the number of persons in each sector, $n_{L}$ and $n_{H}$, we have:

$$
\begin{aligned}
& D_{L}=\frac{n_{H}}{n}\left(y_{H}-y_{L}\right) \\
& A_{H}=\frac{n_{L}}{n}\left(y_{H}-y_{L}\right)
\end{aligned}
$$

As shown in Figure 1, when the modern sector is tiny, $G_{L}$ is close to zero. Almost everyone in the society has the same low income, so that $n_{H}$ and $D_{L}$ are very low. The situation in the modern sector is the opposite. Since almost everyone has much lower income than those in the modern sector, the individual inequality measure there, $G_{H}$ is very high. Now, as development proceeds, $G_{L}$ rises monotonically and $G_{H}$ falls monotonically - - a necessary result in this simple model. ${ }^{12}$ It is interesting to think what this would mean if the personal inequality assessments reflected individual attitudes. People in the traditional sector would believe that inequality was becoming steadily worse while those in the modern sector would think the opposite, hardly a recipe for social harmony.

How does one resolve the conflict when the trend in inequality looks as radically different from the standpoint of two population groups as in the Kuznets transformation? The Gini coefficient offers a solution - - take an average of the personal assessments. Thus in the Kuznets curve example, $G$ is a population weighted average of the values of $G_{L}$ and $G_{H}$. An alternative would be, in effect, to take a vote on the question of whether inequality was rising or falling - - a "democratic" approach. Here the democratic approach, based on personal inequality assessments, would say that inequality rises until $p=$ $1 / 2$ and falls thereafter. In the example, $G$ says that inequality rises until $p=1 / 4$ and falls after that. That is because $G_{H}$ falls faster than $G_{L}$ rises, so that averaging $G_{H}$ and $G_{L}$, even using population weights, places greater relative importance on the decline in $G_{H}$ than on the rise in $G_{L}$.

Our analysis shows that, unfortunately, use of the Gini coefficient could cause confusion during the Kuznets transformation. The Gini begins to fall "too soon". If the behavior of $G$ were used as an input into policy decisions, this could potentially lead to a relaxation of inequality-reducing measures in a country where the majority of the population had yet to join the modern sector and still had rising personal inequality.

The above analysis would not be affected significantly by moving from the $\lambda=1 / 2$ case to $\lambda \neq 1 / 2$. There would of course be no impact on the time path of $G$. Since personal inequality in each sector only depends either on deprivation (in the traditional sector) or advantage (in the modern sector), at the individual level there would simply be a rescaling of $G_{L}^{\lambda}$ and $G_{H}^{\lambda}$ at each point in the Kuznets process. For a majority of people personal inequality would still be rising until $p=1 / 2$ is reached, and above that point the opposite would still be true. $G$ would have its peak at the same point as with $\lambda=1 / 2$. In terms of Figure 1, there would be a proportionate shift of the $G_{L}$ curve by the factor $2(1-\lambda)$ and a shift of the $G_{H}$

\footnotetext{
${ }^{12} D_{L}$ rises with the increase in $n_{H}$ and $A_{H}$ falls as $n_{L}$ declines. But one must also account for $\bar{y}$ rising throughout the Kuznets process when analyzing $G_{L}$ and $G_{H}$. This rise reinforces the decline of $A_{H}$ to ensure that $G_{H}$ must fall throughout. And while the rise in $\bar{y}$, by itself, would make $D_{L}$ fall, the increase in $n_{H}$ has a stronger effect, so that $G_{L}$ rises all through the process.
} 
curve in the opposite direction by the factor $2 \lambda$. In the case where $\lambda<1 / 2$, the $G_{L}$ and $G_{H}$ curves (now $G_{L}^{\lambda}$ and $G_{H}^{\lambda}$ ) would move towards each other, while if $\lambda>1 / 2$ the result would be the opposite.

What difference does it make if incomes are not constant within the two sectors during the Kuznets transformation? The question is whether changes in $\left(y_{H}-y_{L}\right) / \bar{y}$ can reverse those of $\frac{n_{H}}{n}$ or $\frac{n_{L}}{n}$ in the calculations of $G_{H}^{\lambda}$ and $G_{L}^{\lambda}$, respectively. The answer depends on the percentage size of the possibly opposing changes. In the case of China, at least, the income changes appear to have been dominated by population shift. Identifying urban areas as our $H$ sector and rural areas as $L$, from 1980 to $2014\left(y_{H}-\right.$ $\left.y_{L}\right) / \bar{y}$ fell by $18 \%$ in China while $\frac{n_{L}}{n}$ dropped $44 \%$ and $\frac{n_{H}}{n}$ went up $183 \% .{ }^{13}$

\section{Polarization}

There is much theoretical and empirical literature on polarization (including Foster and Wolfson, 1992; Esteban and Ray, 1994; Acemoglu and Autor, 2011; Autor and Dorn, 2013; Green and Sand, 2015). Polarization can take different forms. Without saying so, we have already been discussing one form in the Kuznets transformation, which has two poles: the traditional society and the modern sector. At the starting point, with everyone in the traditional sector, there is no polarization. With population shifting to the modern sector, polarization initially rises, as does aggregate inequality according to the Gini coefficient. Then there is a phase where polarization continues to rise but changes in the Gini coefficient turn from positive to negative. Finally, when the modern sector population becomes a majority, polarization begins to fall, reaching zero when everyone is in the modern sector. In this simple case a democratic assessment based on personal inequality would agree with the polarization analysis.

Polarization in labor markets has received much attention in the US and other high income countries in recent years. In this case the relative demand for labor shifts away from mid-level occupations to both low-skilled and (especially) high skilled occupations. Other things constant this should result in a shift in labor force composition away from the middle toward both the top and bottom. Such a shift has indeed occurred over significant timespans in the US, Canada, the UK, Germany and some other European countries (Acemoglu and Autor, 2011; Green and Sand, 2015). In most cases the wages of highly skilled workers have increased while those of workers in mid-level occupations have tended to decline. In the US it has also been found that wages have risen in certain low skilled occupations (Autor and Dorn, 2013) although that trend has not been seen in some other leading OECD countries (Green and Sand, 2015).

We will analyze the effects of labor market polarization on personal inequality in two steps, first considering only the effects of population shift, that is a rise in the number of individuals at low and high incomes combined with a reduction in the number at middle income. Subsequently we will look at the effect of changes in income. Assume that there are just three income levels in a society and that they display $y_{L}<y_{M}<y_{H}$. Numbers of individuals in the three groups are $n_{L}, n_{M}$, and $n_{H}$. As in the

\footnotetext{
13 The \% changes reported here were calculated using tables 2-1 and 6-6 of National Bureau of Statistics of China (2016), which indicate an urbanization rate of $19.4 \%$ in 1980 and 54.8\% in 2014. Disposable household income per capita in urban areas was 478 yuan in 1980 and 29,381 yuan in 2014; the corresponding rural numbers were 191 and 989 yuan.
} 
Kuznets case the GAPIIs of people in the bottom group and top groups are given by $G_{L}^{\lambda}=\frac{\lambda D_{L}}{\bar{y}}$ and $G_{H}^{\lambda}=$ $\frac{(1-\lambda) A_{H}}{\bar{y}}$.

Once again, qualitative results for $G_{L}^{\lambda}$ and $G_{H}^{\lambda}$ will be the same as for $G_{L}$ and $G_{H}$, so we will focus on the latter for simplicity. The increase in $n_{H}$ due to polarization will tend to make $A_{H}$ and $G_{H}$ decrease since (from 5i):

$$
A_{H}=\frac{\left(n_{L}+n_{M}\right)}{n}\left(y_{H}-\bar{y}_{H}^{l}\right)
$$

However, there is now an offsetting effect because $\bar{y}_{H}^{l}$ falls due to the population shift from the middle to lower groups, and therefore $\left(y_{H}-\bar{y}_{H}^{l}\right)$ increases. It can readily be shown that:

$$
\begin{aligned}
& > \\
\Delta A_{H}, \Delta G_{H} & =0 \text { as } \frac{\Delta n_{L}}{-\Delta n_{M}}=\frac{y_{H}-y_{M}}{y_{H}-y_{L}} \\
& <
\end{aligned}
$$

Now $\frac{y_{H}-y_{M}}{y_{H}-y_{L}}<1$ and $\frac{\Delta n_{L}}{-\Delta N_{M}}<1$ as well, so it is not immediately clear which way the inequality will go. However, we can make a prediction in a "leading case". With a positively skewed distribution of income we would have $\frac{y_{H}-y_{M}}{y_{H}-y_{L}}>\frac{1}{2}$, so that if half or fewer of those leaving the middle income group go to the lower group (which is in line with the experience in the US at least) we have $\frac{\Delta n_{L}}{-\Delta n_{M}}<\frac{1}{2}$ and $A_{H}$ and $G_{H}$ will decline, as in the Kuznets case.

Turning to the bottom group, from (5ii) we have:

$$
D_{L}=\frac{\left(n_{M}+n_{H}\right)}{n}\left(\bar{y}_{L}^{h}-y_{L}\right)
$$

And it can be shown that:

$$
\begin{aligned}
& > \\
\Delta D_{L}, \Delta G_{L} & =0 \text { as } \frac{-\Delta n_{M}}{\Delta n_{H}}=\frac{y_{H}-y_{L}}{y_{M}-y_{L}} \\
& <
\end{aligned}
$$

Now, $\frac{y_{H}-y_{L}}{y_{M}-y_{L}}>1$ and $\frac{-\Delta n_{M}}{\Delta n_{H}}>1$ as well, so again there is ambiguity. However, in the leading case identified above $\frac{y_{H}-y_{L}}{y_{M}-y_{L}}>2$ and $\frac{-\Delta n_{M}}{\Delta n_{H}}<2$, so $D_{L}$ and $G_{L}$ will rise, as in the Kuznets analysis. This would be the result of the increase in $\bar{y}_{L}^{h}$ having a larger effect on $D_{L}$ and $G_{L}$ than the decline in $n_{L}^{h}=\left(n_{M}+\right.$ $\left.n_{H}\right)$.

Population shift has unambiguous results for the middle group because, unlike the case of the top and bottom groups, the income differences vis-à-vis higher or lower groups are not affected by changes in $n_{L}, n_{M}$, and $n_{H} . G_{M}^{\lambda}$ depends on both $A_{M}$ and $D_{M}$,

$$
G_{M}^{\lambda}=\frac{\lambda A_{M}+(1-\lambda) D_{M}}{\bar{y}}
$$


while personal advantage and deprivation are proportional to $n_{L}$ and $n_{H}$ respectively:

$$
\begin{aligned}
& A_{M}=\frac{n_{L}}{n}\left(y_{M}-y_{L}\right) \\
& D_{M}=\frac{n_{H}}{n}\left(y_{H}-y_{M}\right)
\end{aligned}
$$

Since both $n_{L}$ and $n_{H}$ rise in polarization, the pure effect of population shift is for $G_{M}^{\lambda}$ to increase for any value of $\lambda$.

Turning to income changes, as mentioned above, it is typically observed in labor market polarization that $y_{H}$ rises and $y_{M}$ declines. In the US it has also been found that $y_{L}$ rises. The rise of $y_{H}$ opposes the "leading case" effects of population shift found above for the $H$ group, so that personal inequality may rise at the top once income changes are taken into account. Impacts for both middle and lower groups are theoretically ambiguous. With $\left(y_{H}-y_{M}\right)$ rising, $D_{M}$ also rises, tending to make $G_{M}^{\lambda}$ increase. But $A_{M}$ may fall if $\left(y_{M}-y_{L}\right)$ declines sufficiently. (The example considered below shows this can occur in practice.) If $A_{M}$ falls then $G_{M}^{\lambda}$ will also fall if the weight placed on $A_{M}$ in (28) is sufficiently large. Finally, the impact of income changes on $G_{L}^{\lambda}$ is ambiguous since $\left(\bar{y}_{L}^{h}-y_{L}\right)$ in (26) may fall if $y_{L}$ rises sufficiently and also because the fall of $y_{M}$ reduces the change in $\bar{y}_{L}^{h}$, possibly even making it negative.

Given the theoretical ambiguity of the behavior of $G_{L}^{\lambda}, G_{M}^{\lambda}$ and $G_{H}^{\lambda}$ it is helpful to consider a real-world example. Autor and Dorn (2013) set out the changes in employment shares and wage rates for six broad occupational groups in the U.S. from 1980 to 2005. From Table 1, the top group, consisting of managers, professionals, technicians, finance and public safety occupations experienced a $29 \%$ increase in employment share and a 36\% rise in wage rates over those years. The middle four groups together had a $22 \%$ drop in employment share and only a $9 \%$ increase in wages. The bottom group, consisting of service occupations, had a $30 \%$ rise in employment share and a $17 \%$ increase in wages. These changes provide a dramatic example of labor market polarization.

For the sake of this example, assume that everyone within each of the six Autor and Dorn occupational groups has the same income. Using that assumption Table 1 shows $G_{i}$ rising for all six groups, as does deprivation $D_{i}$ (except for the top group, where it is identically zero.) On the other hand, advantage, $A_{i}$, falls for groups 2, 3 and 4, because their wages decline relative to the wage of the bottom group. ${ }^{14}$ These results are obtained with $\lambda=1 / 2$. If $A_{i}$ is weighted sufficiently more heavily, $G_{i}^{\lambda}$ declines from 1980 to 2005 for each of groups 2,3 and 4 . The critical values of $\lambda$ are $0.61,0.73$ and 0.89 for groups 2,3 and 4 respectively. Thus, if these groups were sufficiently more concerned about advantage than deprivation they would regard polarization as having reduced inequality between 1980 and 2005 . While worth noting, this result may not affect one's conclusions much in view of the dominant opinion in the literature that $\lambda \leq 1 / 2$ likely holds for most people.

\footnotetext{
${ }^{14}$ Note that in 2005 group 4 has a lower mean wage than group 5. This is accounted for in the numbers shown in Table 1. In 2005 group 4 only has an advantage over group 6, while group 5 has an advantage over both groups 4 and 6.
} 
Table 1

Advantage $A_{i}$, Deprivation $D_{i}$, and Personal Inequality with $\lambda=1 / 2$, $G_{i}$, by Occupation Group - - Polarization Example Based on US Data, 1980 and 2005

\begin{tabular}{|c|c|c|c|c|c|c|}
\hline Year & $\begin{array}{c}\text { Occupation } \\
\text { Group }\end{array}$ & $\begin{array}{c}\text { Employment } \\
\text { Share }\end{array}$ & $\begin{array}{c}\text { Mean Wage } \\
(2004 \text { \$s })\end{array}$ & $A_{i}$ & $D_{i}$ & $G_{i}$ \\
\hline \multirow{6}{*}{1980} & 1 & 0.316 & 17.0 & 3.415 & 0 & 0.126 \\
\hline & 2 & 0.048 & 15.6 & 2.524 & 0.412 & 0.108 \\
\hline & 3 & 0.216 & 13.6 & 1.224 & 1.156 & 0.088 \\
\hline & 4 & 0.099 & 11.9 & 0.528 & 2.117 & 0.098 \\
\hline & 5 & 0.222 & 11.3 & 0.305 & 2.589 & 0.107 \\
\hline & 6 & 0.099 & 8.2 & 0 & 5.364 & 0.198 \\
\hline \multirow{6}{*}{2005} & 1 & 0.409 & 23.1 & 6.108 & 0 & 0.180 \\
\hline & 2 & 0.030 & 15.2 & 1.425 & 3.241 & 0.137 \\
\hline & 3 & 0.182 & 13.9 & 0.692 & 3.814 & 0.133 \\
\hline & 4 & 0.046 & 12.7 & 0.399 & 4.716 & 0.150 \\
\hline & 5 & 0.204 & 13.5 & 0.537 & 4.069 & 0.135 \\
\hline & 6 & 0.129 & 9.6 & 0 & 7.413 & 0.218 \\
\hline
\end{tabular}

Notes: (i) The mean wage is the geometric mean hourly wage derived from the mean log hourly wage reported by Autor and Dorn (2013), (ii) $G_{i}$ is the personal inequality index $G_{i}^{\lambda}$ when $\lambda=1 / 2$, (iii) the occupational groups are:

1. managers, professionals, technicians, finance and public safety occupations.

2. production and craft occupations

3. transportation, construction, mechanics, mining and farm occupations

4. machine operators and assemblers

5. clerical and retail sales occupations

6. service occupations.

Source: Employment share and mean wage are from Autor and Dorn (2013, Table 1) - - see Note (i). The other columns were calculated by the author. 


\section{Rising Overall Inequality}

In the last four decades, substantial periods of rising overall income inequality, as measured by the Gini coefficient and other conventional indexes, have been observed in a wide range of high income countries (Roine and Waldenström, 2015). In some cases, this reflects polarization, but labelling all increases in inequality as polarization would abuse the latter term. It is probably best to refer to a broadly-based downward movement in the Lorenz curve simply as an increase in inequality.

It is interesting to ask what is likely to happen to personal inequality assessments during a period of rising inequality. Table 2 provides some insight on this question. Using $\lambda=1 / 2$, it shows $G_{i}$ at selected percentiles of lognormal income distributions that have overall $G=0.2,0.3,0.4,0.5$ and 0.6. These Gini values span most of the range observed across countries. For reference, the Gini coefficient for total household income in the U.S. was 0.397 in 1975 and rose with little interruption to 0.479 in 2015 (U.S. Census Bureau, 2016). In the UK, the Gini coefficient for (equivalized) gross household income was 0.300 in 1977 and rose to 0.393 in 2001/02, although it subsequently fell back a little, dropping to 0.358 by 2014/15 (Office for National Statistics, 2016, Figure 9).

Table 2

\section{Personal Inequality Indexes with $\lambda=1 / 2$, by Gini Coefficient and Income Percentile, Lognormal Example}

\begin{tabular}{|c|c|c|c|c|c|}
\hline Gini Coefficient: & $\mathbf{0 . 2}$ & $\mathbf{0 . 3}$ & $\mathbf{0 . 4}$ & $\mathbf{0 . 5}$ & $\mathbf{0 . 6}$ \\
\hline Income Percentile: & & & & & \\
\hline $\mathbf{1}$ & 0.297 & 0.380 & 0.434 & 0.467 & 0.486 \\
\hline $\mathbf{5}$ & 0.244 & 0.328 & 0.392 & 0.437 & 0.468 \\
\hline $\mathbf{1 0}$ & 0.213 & 0.295 & 0.362 & 0.414 & 0.452 \\
\hline $\mathbf{2 5}$ & 0.165 & 0.240 & 0.307 & 0.366 & 0.415 \\
\hline $\mathbf{5 0}$ & 0.140 & 0.208 & 0.272 & 0.331 & 0.385 \\
\hline $\mathbf{7 5}$ & 0.175 & 0.261 & 0.342 & 0.415 & 0.476 \\
\hline $\mathbf{9 0}$ & 0.273 & 0.427 & 0.586 & 0.743 & 0.886 \\
\hline $\mathbf{9 5}$ & 0.362 & 0.591 & 0.850 & 1.135 & 1.431 \\
\hline $\mathbf{9 9}$ & 0.585 & 1.048 & 1.670 & 2.502 & 3.608 \\
\hline
\end{tabular}

Note: $\lambda$ is the weight placed on advantage, $A_{i}$, in the personal inequality index, $G_{i}^{\lambda}$. See text. 
Table 2 shows, first, that $G_{i}$ falls with income up to the median and then rises, as predicted by Proposition 4. The latter increase, from percentile to percentile, rises with income, particularly at the highest levels. We see, for example, with an overall Gini of 0.4 that while $G_{i}$ approximately doubles, from 0.272 to 0.586 , in going from the median to the $90^{\text {th }}$ percentile, it then roughly triples to arrive at 1.670 for the $99^{\text {th }}$ percentile. Second, the table shows that sensitivity to rising inequality is greatest at top income levels. This is more clearly illustrated in Table 3 which shows ratios of $G_{i}$ for pairs of income percentiles, given different values of $G$. The $90: 10 G_{i}$ ratio rises from 1.62 when $G=0.4$ to 1.80 when $G=0.5$, and the 99:1 ratio rises from 3.85 when $G=0.4$ to 5.36 when $G=0.5$. The increase of $G$ by 0.1 , from 0.4 to 0.5 is roughly similar to the rise seen in the U.S. since 1975, and to that in the UK from 1977 to 2001/02, so these results may have real-world relevance. They raise the interesting possibility that high income people could have had the largest increases in personal inequality in recent decades.

Table 3

\section{Ratios of Personal Inequality Indexes with $\lambda=1 / 2$ for Selected Income Percentiles, Lognormal Example}

\begin{tabular}{|c|c|c|c|c|c|}
\hline Gini Coefficient: & $\mathbf{0 . 2}$ & $\mathbf{0 . 3}$ & $\mathbf{0 . 4}$ & $\mathbf{0 . 5}$ & $\mathbf{0 . 6}$ \\
\hline Income Percentiles: & & & & & \\
\hline $\mathbf{6 0 : 4 0}$ & 1.004 & 1.006 & 1.007 & 1.008 & 1.009 \\
\hline $\mathbf{7 0 : 3 0}$ & 1.030 & 1.044 & 1.056 & 1.065 & 1.070 \\
\hline $\mathbf{7 5 : 2 5}$ & 1.059 & 1.087 & 1.112 & 1.132 & 1.146 \\
\hline $\mathbf{8 0 : 2 0}$ & 1.103 & 1.155 & 1.204 & 1.247 & 1.277 \\
\hline $\mathbf{9 0 : 1 0}$ & 1.281 & 1.444 & 1.618 & 1.795 & 1.960 \\
\hline $\mathbf{9 5 : 5}$ & 1.483 & 1.799 & 2.171 & 2.596 & 3.060 \\
\hline $\mathbf{9 9 : 1}$ & 1.968 & 2.757 & 3.850 & 5.361 & 7.430 \\
\hline
\end{tabular}

Note: $\lambda$ is the weight placed on advantage, $A_{i}$, in the personal inequality index, $G_{i}^{\lambda}$. See text.

An idea of the quantitative impact of allowing $\lambda \neq 1 / 2$ is provided in Tables 4 and 5 , which repeat the exercises of Table 2 and 3, but with the range of $G$ confined to [0.3,0.5] and alternate values of $\lambda=0.25$ and 0.75 considered. Note first that $G_{i}^{\lambda}$ initially declines as income rises but hits a minimum at the $(1-\lambda) 100$ th percentile, as predicted by Proposition 4 . Next, we can see that raising $\lambda$ twists the $G_{i}^{\lambda}$ profile. For lower incomes, $G_{i}^{\lambda}$ falls but for higher incomes $G_{i}^{\lambda}$ rises. This means that there is an increase with $\lambda$ in the acceleration of $G_{i}^{\lambda}$ as one goes up the income scale, and a rise in $G_{i}^{\lambda}$ ratios for such income percentile pairs as 90:10 and 99:1 (Table 5). The switch from a negative to positive impact of $\lambda$ on $G_{i}^{\lambda}$ occurs at income percentile 61 for $G=0.3$, at percentile 65 for $G=0.4$, and at percentile 69 for $G=0.5$. 
Table 4

Personal Inequality Indexes with $\lambda=0.25$ and 0.75, by Gini Coefficient and Income Percentile, Lognormal Example

\begin{tabular}{|c|c|c|c|c|c|c|}
\hline & \multicolumn{3}{|c|}{$\lambda=0.25$} & \multicolumn{3}{c|}{$\lambda=0.75$} \\
\hline Gini Coefficient: & $\mathbf{0 . 3}$ & $\mathbf{0 . 4}$ & $\mathbf{0 . 5}$ & $\mathbf{0 . 3}$ & $\mathbf{0 . 4}$ & $\mathbf{0 . 5}$ \\
\hline Income Percentiles: & & & & & & \\
\hline 1 & 0.570 & 0.650 & 0.700 & 0.190 & 0.217 & 0.233 \\
\hline 5 & 0.491 & 0.586 & 0.655 & 0.166 & 0.197 & 0.219 \\
\hline 10 & 0.439 & 0.539 & 0.618 & 0.152 & 0.185 & 0.210 \\
\hline 25 & 0.341 & 0.443 & 0.533 & 0.139 & 0.172 & 0.199 \\
\hline 50 & 0.242 & 0.332 & 0.423 & 0.173 & 0.211 & 0.240 \\
\hline 75 & 0.199 & 0.278 & 0.361 & 0.322 & 0.406 & 0.468 \\
\hline 90 & 0.242 & 0.342 & 0.449 & 0.611 & 0.830 & 1.037 \\
\hline 95 & 0.311 & 0.452 & 0.613 & 0.870 & 1.248 & 1.656 \\
\hline 99 & 0.528 & 0.843 & 1.266 & 1.567 & 2.496 & 3.739 \\
\hline & & & & & & \\
\hline
\end{tabular}

Note: $\lambda$ is the weight placed on advantage, $A_{i}$, in the personal inequality index, $G_{i}^{\lambda}$. See text.

Table 5

Ratios of Personal Inequality Indexes with $\lambda=0.25$ and 0.75 for Selected Income Percentiles, Lognormal Example

\begin{tabular}{|c|c|c|c|c|c|c|}
\hline & \multicolumn{3}{|c|}{$\boldsymbol{\lambda = 0 . 2 5}$} & \multicolumn{3}{c|}{$\boldsymbol{\lambda = 0 . 7 5}$} \\
\hline Gini Coefficient: & $\mathbf{0 . 3}$ & $\mathbf{0 . 4}$ & $\mathbf{0 . 5}$ & $\mathbf{0 . 3}$ & $\mathbf{0 . 4}$ & $\mathbf{0 . 5}$ \\
\hline Income Percentiles: & & & & & & \\
\hline $\mathbf{6 0 : 4 0}$ & 0.787 & 0.811 & 0.837 & 1.406 & 1.401 & 1.382 \\
\hline $\mathbf{7 0 : 3 0}$ & 0.636 & 0.674 & 0.717 & 1.965 & 1.974 & 1.949 \\
\hline $\mathbf{7 5 : 2 5}$ & 0.584 & 0.627 & 0.677 & 2.320 & 2.358 & 2.353 \\
\hline $\mathbf{8 0 : 2 0}$ & 0.549 & 0.599 & 0.656 & 2.747 & 2.847 & 2.897 \\
\hline $\mathbf{9 0 : 1 0}$ & 0.552 & 0.634 & 0.727 & 4.016 & 4.487 & 4.941 \\
\hline $\mathbf{9 5 : 5}$ & 0.633 & 0.772 & 0.936 & 5.252 & 6.329 & 7.548 \\
\hline $\mathbf{9 9 : 1}$ & 0.927 & 1.296 & 1.808 & 8.239 & 11.506 & 16.015 \\
\hline
\end{tabular}

Note: $\lambda$ is the weight placed on advantage, $A_{i}$, in the personal inequality index, $G_{i}^{\lambda}$. See text. 


\section{Discussion and Conclusion}

The fact that the Gini coefficient is the average of "Gini admissible" personal inequality indexes or GAPIIs produces interesting insights. One important feature is that GAPIIs are completely insensitive to transfers of income that occur only among people who have incomes above those of the reference individual, or among those with incomes below. This means that GAPIIs do not obey the Pigou-Dalton principle of transfers. But they do regard transfers from those in the group above the individual to those in the group below as equalizing and transfers in the other direction as disequalizing. These properties explain why the Gini coefficient's sensitivity to transfers depends critically on the number of people with incomes between those of the donor and recipient, which makes it most sensitive in practice to changes in the middle of typical income distributions. This dependence reflects the fact that, aside from the donor and recipient themselves, it is only people between them whose GAPIIs are affected by the transfer.

As we have seen, each GAPII is a weighted average of an individual's deprivation and advantage. That the relative weights placed on these components can vary has a range of implications. For example, the weights could vary across societies, perhaps reflecting differences in individual attitudes. At one extreme all the weight could be placed on deprivation in a society where people resented others being better off than themselves but had no concern about the income of those below them. At the other extreme all the weight could be put on advantage if everyone had been taught to have concern for the "less fortunate" and not to envy the better-off. And, of course, any weighting between these extremes could occur. But in each society, taking the average of GAPII values would still yield the Gini coefficient, since it is unaffected by the relative weight placed on deprivation vs. advantage. So, societies with quite different views about inequality at the individual level, could still all embrace the Gini coefficient as their aggregate measure of inequality. It is tempting to imagine that this might help to explain the wide international popularity of the Gini coefficient.

We have also discussed how personal inequality assessments may behave during secular change in income distribution. In the development context, in the simplest model of the Kuznets transformation, personal inequality for those in the traditional sector rises throughout, while the opposite occurs in the modern sector. If personal inequality reflects individual attitudes, the resulting scope for misunderstanding and conflict seems large. This may throw some light on the tensions that are observed during periods of rapid modernization. A further insight comes from the fact that the Gini coefficient says the Kuznets process stops being disequalizing well before half the population is in the modern sector. Thus, the direction of change in the Gini coefficient may not always reflect majority opinion.

Under polarization, population shifts not only to the top but also to the bottom, with a shrinking middle group. Income tends to rise at the top, fall in the middle, and may rise little at the bottom. In a leading case, population shifts increase personal inequality at the bottom and reduce it at the top, echoing the Kuznets transformation results. Personal inequality rises in the middle if there are no income changes. When income changes are also taken into account it is theoretically possible for any of the population shift effects to be reversed. Given this ambiguity we turned to the real world for some guidance. In an example based on the polarization seen in the US between 1980 and 2005, personal inequality rose for all groups when advantage and deprivation were equally weighted. For three middle groups personal inequality would have fallen if sufficiently more weight were placed on their advantage rather than their deprivation. However, if personal inequality reflects individual attitudes, it seems unlikely that the 
middle groups would indeed have regarded inequality as falling, given the broad consensus in the literature that most people tend to be more concerned about deprivation than advantage.

Finally, the impact of increased dispersion on personal inequality when income is lognormal was examined. This may give an idea of how a broad-based rise in inequality affects personal assessments. Such a trend raises personal inequality at all levels of income irrespective of the relative weights placed on deprivation and advantage. However, it does not do so equally. The increase in personal inequality tends to be greatest for those with the highest incomes. 


\section{References}

Acemoglu, D. and D.H. Autor (2011), "Skills, tasks and technologies: Implications for employment and earnings", Handbook of Labor Economics 4: 1043-1171.

Autor, David H. and David Dorn (2013), "The Growth of Low-Skill Service Jobs and the Polarization of the US Labor Market", American Economic Review 103 (5): 1553-1597.

Clark, Andrew E. and Conchita D’Ambrosio (2015), “Attitudes to Income Inequality: Experimental and Survey Evidence", chapter 13 in Anthony B. Atkinson and François Bourguignon, Handbook of Income Distribution Vol. 2a, $1^{\text {st }}$ edition, North-Holland Elsevier: Amsterdam, 1147-1208.

Cojocaru, A. (2014), "Fairness and inequality tolerance: evidence from the Life in Transition survey", Journal of Comparative Economics 42 (3): 590-608.

Cowell, Frank A. (2011), Measuring Inequality, $3^{\text {rd }}$ edition, Oxford: Oxford University Press.

Dalton, Hugh (1920), “The Measurement of Inequality of Incomes”, Economic Journal 30: 348-361.

D’Ambrosio, Conchita and J.R. Frick (2007), "Income Satisfaction and Relative Deprivation: an Empirical Link”, Social Indicators Research 81: 497-519.

D’Ambrosio, Conchita and J.R. Frick (2012), "Individual Well-Being in a Dynamic Perspective", Economica 79: 284-302.

Esteban, J. and D. Ray (1994), “On the measurement of polarization”, Econometrica 62: 819-851.

Fehr, Ernst and Klaus M. Schmidt (1999), “A Theory of Fairness, Competition and Cooperation”, Quarterly Journal of Economics 114: 817-868.

Fehr, Ernst and Klaus M. Schmidt (2003), "Theories of Fairness and Reciprocity: Evidence and Economic Applications", in Dewatripoint, M. L.P. Hansen, and S.J. Turnovsky (eds.), Advances in Economic Theory, Eighth World Congress of the Econometric Society, vol. 1, Cambridge University Press: Cambridge: 208-257.

Foster, James E. and Michael C. Wolfson (1992), "Polarization and the decline of the middle class", mimeo, reprinted 2010 in Journal of Economic Inequality 8: 247-273.

Gini, Corrado (1914), "On the measurement and variability of characters", METRON-International Journal of Statistics, LXIII (Part II): 3-38.

Green, David A. and Benjamin M. Sand (2015), “Has the Canadian labour market polarized?", Canadian Journal of Economics 48 (2): 621-646.

Knight, John (2014), “Inequality in China: An Overview”, The World Bank Research Observer 29 (1): 119. 
Kuznets, Simon (1955), "Economic Growth and Income Inequality", American Economic Review 45: 128.

Li, Shi and Terry Sicular (2014), "The Distribution of Household Income in China:

Inequality, Poverty and Policies”, The China Quarterly 217 (March): 1-41.

National Bureau of Statistics of China (2016), China Statistical Yearbook - 2015, www.stats.gov.cn accessed Jan. 2, 2016.

Office for National Statistics (2016), "The Effects of Taxes and Benefits on Household Income, Financial Year Ending 2015", Government of the United Kingdom.

http://www.ons.gov.uk/ons/datasets-and-

tables/index.html?pageSize $=50 \&$ sortBy $=$ none \&sortDirection=none\&newquery $=$ effects + of + taxes + and + be nefits\&content-type=Reference+table\&content-type=Dataset Accessed Dec. 11, 2016.

Qi, Liyan (2015), "Wealth Gaps Top List of Concerns Ahead of China's Political Meetings", Wall Street Journal: China, February 27. http://blogs.wsj.com/chinarealtime/2015/02/27/wealth-gap-tops-list-ofconcerns-ahead-of-chinas-political-meetings/ Accessed January 20, 2016.

Roine, Jesper and Daniel Waldenström (2015), "Long-Run Trends in the Distribution of Income and Wealth", chapter 7 in Anthony B. Atkinson and François Bourguignon, Handbook of Income Distribution Vol. $2 a, 1^{\text {st }}$ edition, North-Holland Elsevier: Amsterdam, 469-492.

Runciman, W.G. (1966), Relative Deprivation and Social Justice, London: Routledge and Kegan Paul.

Sen, Amartya (1973), On Economic Inequality, London: Oxford University Press (Clarendon).

Sicular, Terry (2013), "The Challenge of High Inequality in China", Inequality in Focus 2(2): 1-8. http://www.worldbank.org/content/dam/Worldbank/document/Poverty\%20documents/Inequality-InFocus-0813.pdf

Teyssier, Sabrina (2012), "Inequity and risk aversion in sequential public good games", Public Choice 51 (1-2): 91-119.

Yitzhaki, Shlomo (1979), "Relative Deprivation and the Gini Coefficient", Quarterly Journal of Economics, 93 (2): 321-324.

Yitzhaki, Shlomo (1982), "Relative Deprivation and Economic Welfare". European Economic Review 17: 99-113.

Yitzhaki, Shlomo (1998), "More than a Dozen Alternative Ways of Spelling Gini", Research on Economic Inequality 8: 13-30.

U.S. Census Bureau (2016), "Historical Income Tables: Income Inequality", http://www.census.gov/data/tables/time-series/demo/income-poverty/historical-income-inequality.html Accessed Dec. 11, 2016. 


\section{Appendix}

This appendix provides proofs of propositions 4 and 5 .

Proposition 4: If $y_{1}<y_{2}<\cdots<y_{n}$,

$$
\begin{aligned}
G_{i+1}^{\lambda} & =G_{i}^{\lambda} \text { as } \quad \frac{i}{n}=1-\lambda \\
< & <
\end{aligned}
$$

Proof: From (4'), (5) and (22), and using the assumption that $y_{1}<y_{2}<\cdots<y_{n}, G_{i+1}^{\lambda}=G_{i}^{\lambda}$ as:

$$
\lambda \sum_{j=1}^{i}\left(y_{i+1}-y_{j}\right)+(1-\lambda) \sum_{j=i+2}^{n}\left(y_{j}-y_{i+1}\right) \stackrel{>}{=} \lambda \sum_{j=1}^{i-1}\left(y_{i}-y_{j}\right)+(1-\lambda) \sum_{j=i+1}^{n}\left(y_{j}-y_{i}\right)
$$

Now, the left-hand side of this expression can be written:

$$
\lambda\left[\sum_{j=1}^{i-1}\left(y_{i}-y_{j}\right)+i\left(y_{i+1}-y_{i}\right)\right]+(1-\lambda)\left[\sum_{j=i+1}^{n}\left(y_{j}-y_{i}\right)+(n-i)\left(y_{i}-y_{i+1}\right)\right]
$$

Hence, (A1) simplifies to:

$$
\begin{aligned}
\lambda i\left(y_{i+1}-y_{i}\right)+(1-\lambda)(n-i)\left(y_{i}-y_{i+1}\right) & >0 \\
& <
\end{aligned}
$$

which is equivalent to:

$$
\begin{aligned}
\lambda i-(1-\lambda)(n-i) & >0 \\
& <
\end{aligned}
$$

which becomes:

$$
\begin{aligned}
& > \\
\lambda n+i-n & =0 \\
& <
\end{aligned}
$$

from which one readily derives the result that

$$
\begin{aligned}
G_{i+1}^{\lambda} & >G_{i}^{\lambda} \text { as } \quad \frac{i}{n}=1-\lambda \\
< &
\end{aligned}
$$


Proposition 5: If $y_{1}<y_{2}<\cdots<y_{n}$

(i) $G_{1}^{\lambda}=(1-\lambda)\left(1-\frac{y_{1}}{\bar{y}}\right)$

(ii) if $n$ is odd, $G_{m e d}^{\lambda}=\frac{n-1}{2 n \bar{y}}\left[(1-\lambda) \bar{y}_{m e d}^{h}-\lambda \bar{y}_{m e d}^{l}\right]$; if $n$ is even, $G_{m e d}^{\lambda}$ is not defined,

(iii) $G_{n}^{\lambda}=\lambda\left(\frac{y_{n}}{\bar{y}}-1\right)$

Proof: (i) From (5) and (22), given that $n_{1}^{l}=0$,

$$
\begin{aligned}
G_{1}^{\lambda} & =\frac{(1-\lambda)}{n \bar{y}}\left[n_{1}^{h}\left(\bar{y}_{1}^{h}-y_{1}\right)\right] \\
& =\frac{(1-\lambda)}{n \bar{y}}\left[\sum_{j=2}^{n} y_{j}-(n-1) y_{1}\right] \\
& =\frac{(1-\lambda)}{n \bar{y}}\left(\sum_{j=1}^{n} y_{j}-n y_{1}\right) \\
& =\frac{(1-\lambda)}{n \bar{y}}\left(n \bar{y}-n y_{1}\right) \\
& =(1-\lambda)\left(1-\frac{y_{1}}{\bar{y}}\right)
\end{aligned}
$$

(ii) From (5) and (22), if $n$ is odd we have:

$$
G_{m e d}^{\lambda}=\frac{\lambda}{n \bar{y}} n_{m e d}^{l}\left(y_{m e d}-\bar{y}_{m e d}^{l}\right)+\frac{(1-\lambda)}{n \bar{y}} n_{m e d}^{h}\left(\bar{y}_{m e d}^{h}-y_{m e d}\right)
$$

Noting that $n_{\text {med }}^{l}=n_{\text {med }}^{h}=\frac{n-1}{2}$,

$$
G_{m e d}^{\lambda}=\frac{n-1}{2 n \bar{y}}\left[(1-\lambda) \bar{y}_{m e d}^{h}-\lambda \bar{y}_{m e d}^{l}\right]
$$

If $n$ is even there is no individual with median income since $y_{1}<y_{2}<\cdots<y_{n}$.

(iii) From (5) and (22), given that $n_{n}^{h}=0$,

$$
\begin{aligned}
G_{n} & =\frac{\lambda}{n \bar{y}}\left[n_{n}^{l}\left(y_{n}-\bar{y}_{n}^{l}\right)\right] \\
& =\frac{1}{n \bar{y}}\left[(n-1) y_{n}-\sum_{j=1}^{n-1} y_{j}\right]
\end{aligned}
$$




$$
\begin{aligned}
& =\frac{\lambda}{n \bar{y}}\left[n y_{n}-\sum_{j=1}^{n} y_{j}\right] \\
& =\frac{\lambda}{n \bar{y}}\left[n y_{n}-n \bar{y}\right] \\
& =\lambda\left[\frac{y_{n}}{\bar{y}}-1\right]
\end{aligned}
$$

